\title{
Improving Reverse Logistics Processes Using Item-level Product Lifecycle Management
}

\author{
Carl Hans, Karl A. Hribernik, Klaus-Dieter Thoben
}

BIBA - Bremer Institut für Produktion und Logistik GmbH

Hochschulring 20, 28359 Bremen, Germany

Fax: +49 4212185610 Tel: +49 4212185512

\{han, hri, tho\}@biba.uni-bremen.de

\begin{abstract}
Sustainability is a key issue for companies offering products and services on the global market. The ever-increasing worldwide demand for raw materials in combination with the rising costs for materials and energy challenges companies to make their products, processes and services more sustainable. More and more customers are demanding sustainable products and services due to their increased awareness about environmental protection. By providing access to data, information and knowledge about products and services the concept of Product Lifecycle Management (PLM) can be applied to reverse logistics processes to improve sustainability. The term PLM and therefore the functionality of existing PLM systems must be considered as quite different. The paper introduces the concept of item-level Product Lifecycle Management. It investigates the requirements that item-level PLM systems must fulfill in order to support sustainability in reverse logistics processes in an appropriate manner. Existing item-level PLM solutions are then investigated according their suitability in the field of reverse logistics.
\end{abstract}

Keywords: Item-Level Product Lifecycle Management, architecture, integration, item-level, sustainability, reverse logistics

\section{Introduction}

Sustainability and consequently reverse logistics is a key issue for companies offering products and services on the global market. The ever-increasing worldwide demand for raw materials in combination with the rising costs for materials and energy challenges companies to make their products, processes and services more sustainable. More and more customers are demanding sustainable products and services due to their increased awareness about environmental protection. Thus, sustainability has also become a significant marketing instrument, attracting customers using terms such as "Green Production". Consequently, approaches to enhance reverse logistics processes garner increasing interest by industry. Additionally, European Community legislation such as Directives 2002/95/EC, 2002/95/EC or EU/2000/53 force manufacturers to establish organizational concepts which are supported by suitable technological infrastructures to ensure the profitability of affected products.

Many different technical as well as non-technical approaches address reverse logistics processes. One which has raised significant industry attention is the concept of Product Lifecycle Management (PLM). PLM is a term used to describe the management of the entire lifecycle of a product, from its conception, through its design and manufacturing and its service to its disposal. Central to the idea is to acquire and maintain data, information and knowledge about services and products which can be used to improve sustainability. Traditionally, PLM systems focus on classes of products and variants rather than individual items. Furthermore, they mostly address the initial phases of the lifecycle; the beginning of life (BOL). They offer document, workflow and project management functionality. This approach renders their potential for enhancing reverse logistics processes limited from the perspective of the seamless availability of data, information and knowledge from the entire product lifecycle, including the middle (MOL) and end of life (EOL) phases.

In contrast to traditional PLM, item-level PLM takes the entire lifecycle of an individual instance of a product or service (item) into account, beginning with its conceptualization and manufacturing, over its usage to its recycling or disposal. Thus item-level PLM can be seen as an extension of traditional PLM which consolidates the class- as well as instance-based view on products and services under one umbrella. Typical examples of how item-level PLM is applied in industry today cover predictive and intelligent maintenance (Schuh, et al. 2005), design for X (Kiritsis, Bufardi und Xirouchakis 2003) or recycling. All of these application areas require a seamless access to data, information and knowledge related to certain instances of products or services. 
Although item-level PLM has already been adopted by industry in a number of very specific sectors, its adoption is not yet widespread nor is its potential for improving sustainability fully exploited. Whilst the technology required is already available, other relevant issues are still subject of research. Semantic modelling of items, management of decentralized data and information sources or issues addressing item-level PLM to support inter-organizational cooperation are examples of current research topics.

\section{Item-level Product Lifecycle Management}

As outlined above, traditional PLM systems tend to focus on the management of information regarding not the product as an individual item but as a marketable commodity. Even more restrictively, their perspective is often limited to the beginning of life of the product in question. This perspective is essentially due to their historical development from systems which were originally designed to support design, development and production tasks. The genealogy of PLM systems can be traced back to CAD/CAM and CIM systems via Product Data Management (PDM) and Product Information Management (PIM) systems.

\subsection{Product Data Management}

Product Data Management (PDM) is a concept for the structuring, storing, managing and providing access to data and documents created throughout a product's development. It deals with data regarding the product's definition, representation and presentation.

PDM was introduced due to the need to manage the steadily increasing amount of data and documents in product development, first and foremost resulting from the adoption of CAD systems. PDM systems implement standards such as ISO 10303, "Industrial automation systems and integration - Product data representation and exchange”, commonly known as STEP, "Standard Exchange of Product Model Data” (Fowler 1995).

The objective of PDM is to enhance the quality and efficiency of product development processes; put simply, to save time and money. Consequently, PDM systems are required to be capable of reproducing the state of a product's data at any point within its development process (Hribernik, et al. 2008).

According to (Abramovici and Sieg 2001), classic PDM functionality encompasses object, component and document management, classification and search functionality, change management and tools for system administration and configuration. However, requirements towards PDM systems often call for support beyond the production phase as well as for cross-enterprise integration and cooperation.

\subsection{Product Lifecycle Management}

PLM is commonly understood as a concept which "seeks to extend the reach of PDM [...] beyond design and manufacturing into other areas like marketing, sale and after sale service, and at the same time addresses all the stakeholders of the product throughout its lifecycle" (Ameri and Dutta 2005).

Contrary to this common understanding, PLM systems are often not adequate solutions for the management of item-specific product information in the usage phase and beyond. This is due to the fundamental changes in the context of the product and the resulting consequences for data management approaches. During the conception and the production phase, only a few stakeholders are involved in the management of product data. These either belong to a single organisation or are tightly coupled to the IT landscape prescribed by the main stakeholder. Responsibility for the product lies with the manufacturer, who also grants physical access to it. The data models and management systems used serve a limited amount of well-known tasks and objectives which are dictated by the manufacturer coordinating the PLM network.

This constellation dramatically changes on moving into the usage phase of the product lifecycle. The foremost difference is that the amount of stakeholders involved in the product lifecycle is no longer static or limited to the networked involved in its conception and production. Rather, the amount of different stakeholders continuously increases throughout the product's life span - owners, operators, service providers and other actors become involved with the product.

At the same time, the clear objectives for product data management visible in the initial lifecycle phase become blurred once the manufacturer renunciates his responsibility for the coordination of the PLM network and the product itself on entering the usage phase. (Jun, Kiritsis and Xirouchakis 2007) Once the product is in the possession of the customer, it becomes difficult for the original manufacturer to keep control of it (Stark 2004).

On top of this, the perspective on the term product shifts after beginning of life from a design with variants towards the individual, physical item.

The consequences of the differences described here between traditional, conception and production focused PLM and a holistic view over the entire lifecycle from the perspective of the individual item are significant from 
a data modelling, data acquisition and data management perspective. For this reason, existing data and information management approaches and systems for the support of traditional PLM are insufficient.

With the introduction of item-level product information management approaches such as the EPCglobal Architecture Framework (EPCGlobal Inc. 2007b), ID@URI (Kärkkäinen, Främling and Ala-Risku 2002) or WWAI (Främling, Harrison and Brusey 2007) and the underlying item-level, auto-ID, product embedded information device (PEID) (Jun, Kiritsis and Xirouchakis 2007) and Intelligent Product (Wong, et al. 2002) (Kärkkäinen, et al. 2003) (Ventä 2007) technologies, the development of suitable IT solution environments for item-level PLM is becoming a reality. A prominent example of such an environment is called closed-loop PLM (Jun, Kiritsis and Xirouchakis 2007). This environment utilises PEIDs based, for example, on RFID or embedded systems and sensor networks to gather lifecycle data for individual products, with a focus on enabling the feedback of product information to each product lifecycle phase. With regards to sustainability and end of life information requirements, both the information flow from previous lifecycle phases into end of life processes as well as back to previous phases can be handled with this approach.

\section{Research Scope}

In order to obtain a better understanding of the key issues item-level PLM is required to address in order to improve the sustainability of products, a number of case studies were carried out in the reverse logistics sector of different types of product.

The scenarios aimed to identify both the similarities in and differences between the requirements set by the types of product studied, allowing an initial set of requirements to be derived from which a contribution to the identification of generic item-level PLM functionality may be made.

The focus of the case studies was laid on the end of life of individual products, more specifically on recycling processes. The key questions in all studies was what contribution the management of item-level PLM information could provide to both optimise the recycling processes themselves and also to create a positive effect on processes in other lifecycle phases. Consequently, whilst the focus of the studies is from the specific perspective of end of life processes, their impact and feedback upon both previous and consequent processes is taken into consideration.

The following sections describe the case studies carried out in the context of this research.

\subsection{Recycling of Electronics Products}

The introduction of the European Community directive 2002/96/EC on Waste Electrical and Electronic Equipment Directive (WEEE Directive) alongside the Restriction of Hazardous Substances Directive (RoHS Directive) 2002/95/EC force manufacturers to act with regards to lifecycle management in order to retain the profitability of these products. The directives, which were adopted into European Law in February 2003, oblige the EU member states to transpose their provisions into national law by August 2004. In Germany, for example, both directives have been implemented as national law (Elektro- und Elektronikgerätegesetz - ElektroG), which regulates the restriction of hazardous substances and the end-of-life treatment for electrical and electronic equipment.

A product was chosen which exemplifies PLM challenges along the lifecycle of electronic products: A computing device consisting of distinct electronic sub-components - a chassis upon which a mainboard, processor, ISDN modem, power supply, display and hard drive are mounted. Each component needs to be dealt with separately at the end of the product's life. This dictates an individual tracking and tracing of each component throughout the entire product lifecycle, that is, item-level PLM. In the other lifecycle phases, the aggregation of the individual components comprising the main product needs to be taken into account, in order to support parts replacement and maintenance in the middle of the product's lifecycle. 


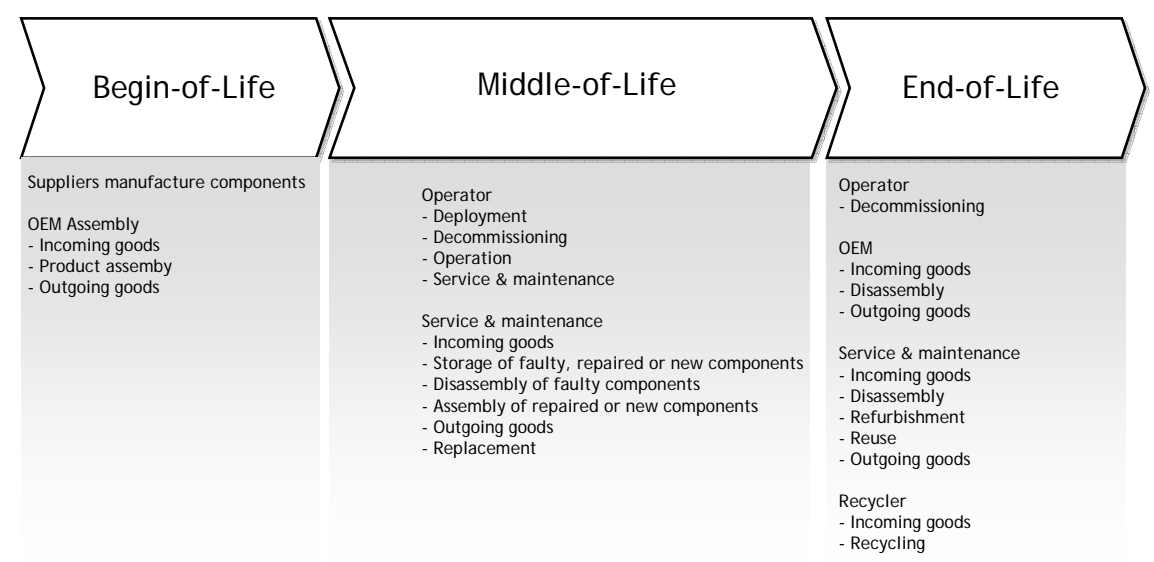

Figure 1: Lifecycle of an Electronic Product (Hribernik, et al. 2007)

Figure 1 illustrates the dynamic cooperation across organizational borders taking place throughout the product's lifecycle. As shown in (Hribernik, et al. 2007), the overall unit is commissioned by an Original Equipment Manufacturer (OEM). Each of the components is manufactured by different enterprises, and then the product is assembled at a subcontracted assembly plant, and deployed and maintained by the operator and subcontractors. Finally, according to the WEEE requirements, the product needs to be taken back by the OEM or a subcontracted disposal service provider, dismantled, and the component parts recycled, reused or refurbished respectively. It follows that any tracking, tracing and information management of the product along with its component parts needs to take place in a standardized, flexible and platform independent manner.

\subsection{Recycling of Automotive Parts}

The ELV (End of Life Vehicle) directive (EU/2000/53) introduced by the EU in 2000 addresses the pollution caused by vehicles which have reached the end of their lives. According to that directive, this scenario was selected to analyse how item-level PLM may be applied to achieve more sustainable automotive products via the substitution of multi-component structures by single-component thermoplastic sandwich-structures in the production of automotive polymer parts.

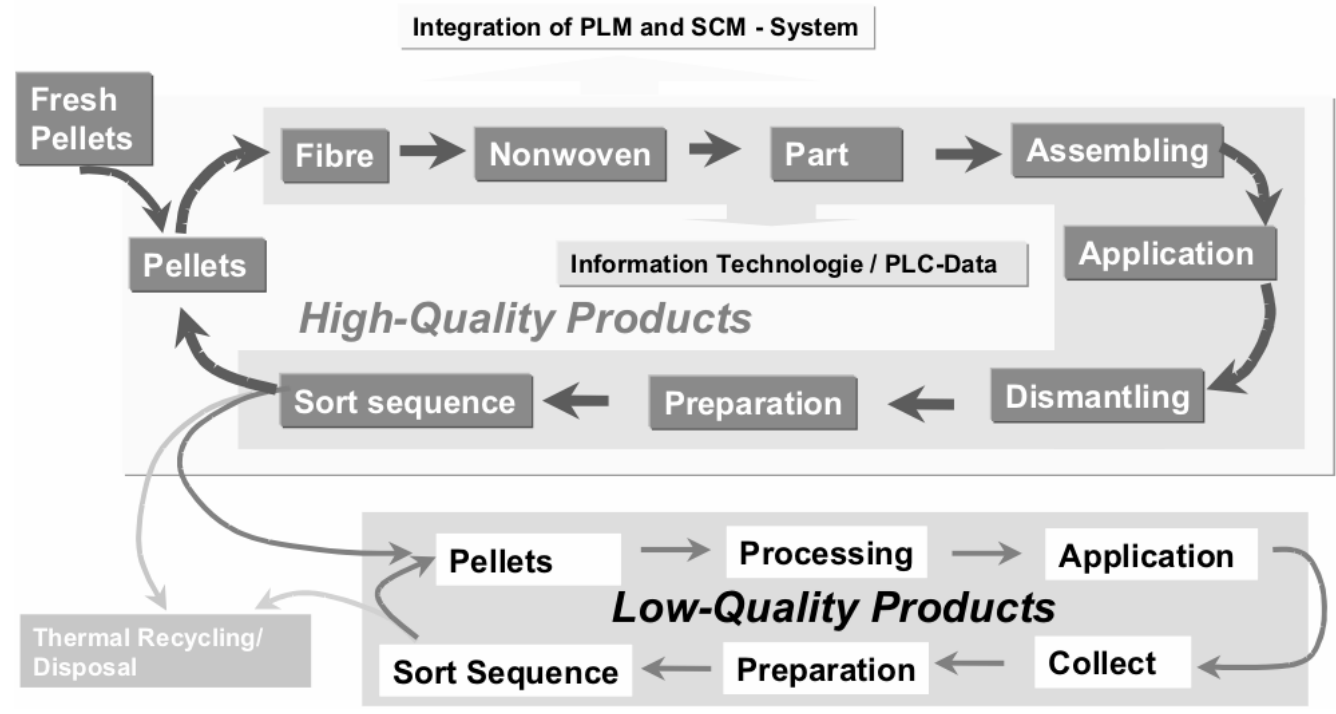

As illustrated in

Figure 2: Controlled Closed Loop Recycling

Figure 2 above, a new state of the art may be defined by establishing an advanced recycling model called Controlled Closed Loop Recycling (CCLR) geared towards 100\% material recovery and remanufacturing of automotive polymer parts replacing material of currently non-recyclable parts with polyethylene (PET) or polypropylene (PP) (Kahn, et al. 2006).

In order to organize the introduced closed loop for polymer automotive parts an item-level PLM system was implemented. For this purpose a universal solution for the identification and tracking of automotive parts was required to enable an automated identification, sorting, classification and routing during dismantling as well as the access to and update of product specific information. A distributed architecture made the collaboration in 
CCLR network to one of the central issues. The IT system had to provide access to specific information like quality, material specifics, dismantling manuals, etc. of automotive items produced by different manufacturers, organize Supply Chain Planning (SCP) and provide notification for the achieved degree of recycling for a car manufacturer in the end of life phase of a vehicle in order to fulfil the ELV directive.

\subsection{Recycling of Plastics Products}

Figure 3 below shows a typical application scenario in which major benefits can be achieved by applying item-level PLM. It demonstrates the lifecycle of plastic component parts which are to be recycled. Their end of life is initiated after the dismantling of the respective products. After dismantling the assortment of individual components are delivered to a point of collection (for example, a container).

Milling commences when a sufficient amount of plastic components is available. Before that, a manual or automated sorting system sorts the components for recycling from the container. After milling and filling the material is sent to another recycling facility by truck. There, the material is put into storage. This facility produces new plastic granulates on customer demand, which in turn become the basis for new plastic products. This process signifies the transition of the EOL phase of one product into the BOL phase of another.

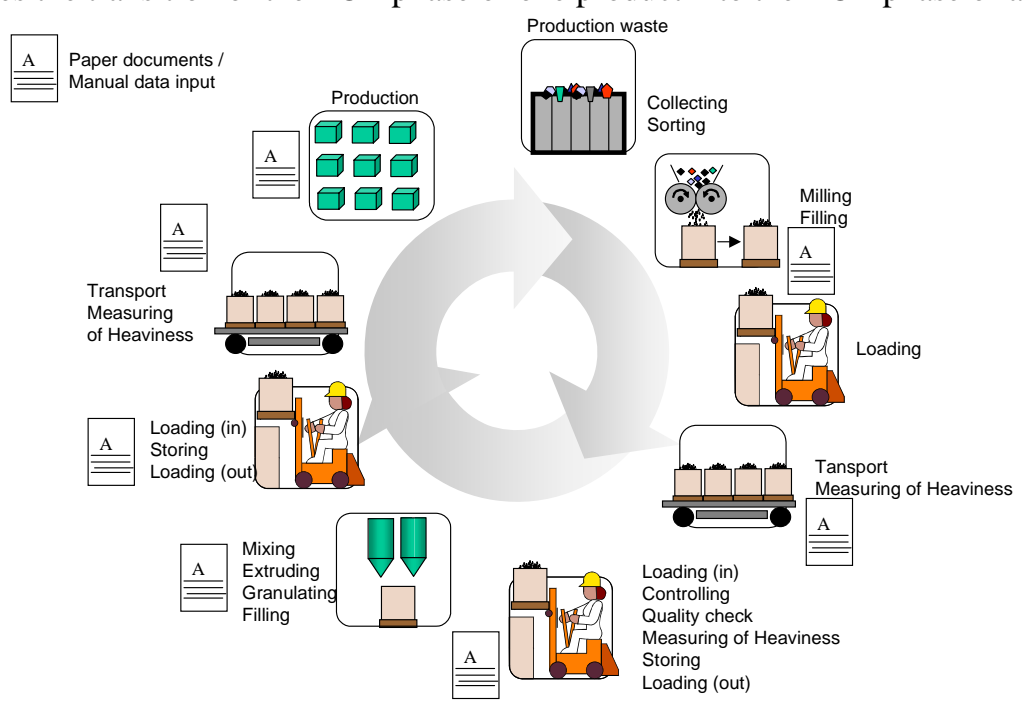

Figure 3: The End-of-Life Phase of Plastic Components (Hribernik, et al. 2007)

In reverse logistics of plastic products, three major goals have to be achieved. The first goal is cost optimization of the reverse logistic material flow, the second the quality level of the logistic service units and the third maintaining the flexibility and adaptability of the reverse logistics systems.

In a closed loop customers have to be served on both the input and output sides (Figure 4). This means that both sides are market driven and dependent on approaches which can react in time, can flexibly react to changes in the market and are reliable in terms of delivery in time and material quality. If this can be assured, the inclusion of raw materials can be reduced to a minimum (by the reduced mixing of raw materials with recycled materials for improving the quality).

The information available and its management is currently less developed in reverse logistics to, for example, distribution logistics. Little information is available about the products handled in terms of their material composition, usage history and modification made to the product throughout their lifecycle. The collection systems' information management is often paper-based and transport containers identified with paper labels only. The consequence of this is data loss and inaccuracy, leading to inefficient and unsustainable processes. These weaknesses make the application an of item-level PLM approach an attractive proposition for the optimization of these processes in the end of life of plastic components. 


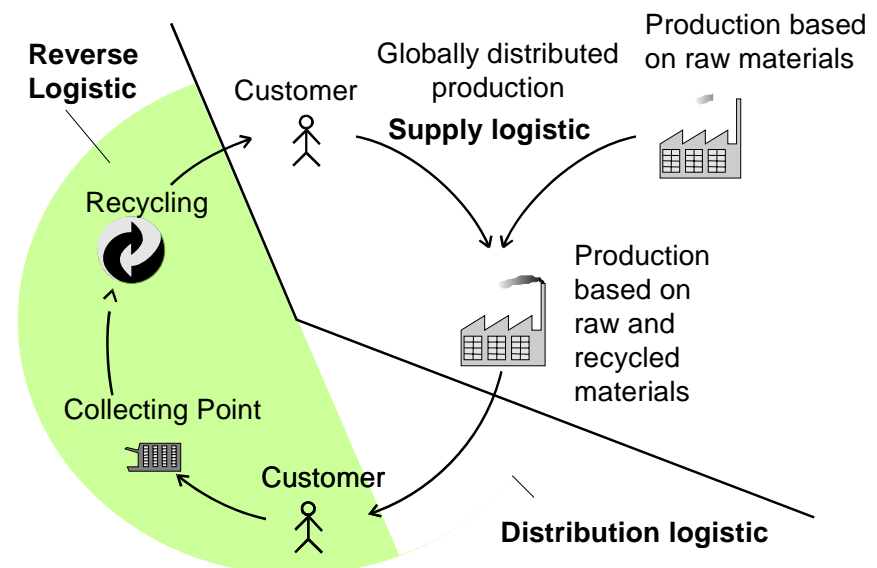

Figure 4: Closed Loop Reverse Logistics System in Plastic Recycling

\section{Application of Item-level PLM Systems to Support Reverse Logistics Processes}

All three business cases depicted above show the potential of item-level PLM to improve reverse logistics processes. However, item-level PLM systems are very different regarding their functionalities as well as the application fields they address. An assessment of item-level PLM systems therefore requires a requirements catalogue which is reflecting generic functionalities needed to support reverse logistics processes. These requirements are discussed in the following by analysing the business cases presented above.

The MOL and EOL phases of the lifecycle can benefit most from the introduction of item-level PLM approaches. During the utilisation of a product item-level data and information could be applied to offer additional services which are tailored to the individual needs of the owner. Due to the availability of item-level data new services can be much more sophisticated than other solutions such as Location Based Services. Another example of how item-level data can be exploited is the field of maintenance. As an example concept Predictive Maintenance (PM) can be mentioned here. Regarding sustainability PM allows for instance a selective exchange of only those parts of a product that needs to be exchanged in order to ensure its operation.

During the EOL phase, item-level information can be used to support decisions on the further treatment of individual products. Today recycling still lacks this kind of data. Consequently, the amount of products recycled or refurbished is not as high as it should be. Access to item-level data can significantly increase this amount as products as well as their components then can be treated individually based on data representing their state (e.g. operation time or material composition).

In order to distinguish different items from each other, a unified, unique item-level identification is required. The ability to refer to individual items is a basic necessity for item-level PLM systems. This identification forms the link between items and data associated with them.

All business cases illustrate that various stakeholders in addition to the owner are involved in the product lifecycle. Thus, item-level data is generated at more than one physical location by a number of heterogeneous systems. Therefore, item-level PLM needs to support the decentralised management of item-level data. Relevant issues to be tackled in this context include the identification of data sources containing relevant data, the correct interpretation of data taken from unknown sources as well as the integration of data from different sources into logical views.

An item-level PLM system must also provide generic interfaces to access item-level data. These interfaces are the basis of an open environment which allows the connection of external IT-systems through open interfaces. In order to establish reliable and robust communication of item-level data between all of the entities within a decentralised system, an asynchronous messaging concept for the storage and retrieval of item-level data is necessary. Asynchronous messaging rids an entity which is requesting data from waiting until an answer arrives. At the same time it reflects normal situations in distributed systems such as temporarily unavailability of system components.

A further requirement is a mechanism for the automatic communication of item-level state changes. This may be realised by subscription mechanisms which require an entity interested in certain data to express its interest (subscribe) in the data required. Then, state changes of individual items are indicated to the subscribers as soon as the associated item-level is modified.

A further requirement stemming from the involvement of multiple stakeholders in the product lifecycle is for adequate security mechanisms to protect item-level data from being manipulated or misused. This requirement 
addresses both communication as well as protection against the duplication of item-level references. From manufacturers perspective the protection of product data and knowledge is considered as a key issue.

Usually, each party involved in the lifecycle of a product uses its own syntax and semantics of item-level data. Apart from those data structures which are standardized (e.g. Electronic Product Code) data is usually specified in a proprietary way along internal company-specific data standards. Using item-level data over the whole lifecycle of a product means to use it across company borders. This requires a common understanding of the syntax and semantic of data. Offering a unified approach for item-level data modelling which can be adopted by all stakeholders would considerably simplify the problem of data integration.

Until here, item-level PLM was mainly considered from the perspective of item-level data. However, highest value can be achieved from item-level PLM solutions which comprises concepts for the transmission of decentralised data into information and knowledge. This process can be considered as the purposeful processing or transformation of data in order to address very specific questions. An example for such a problem covers the redesign or improvement of a product based on the analysis of field data which could be provided by an item-level PLM system. Getting the right conclusions could be considerably supported by generating information or knowledge out of existing item-level data. The requirements identified in the above sections are summarized in Table 1 below.

\begin{tabular}{|l|}
\hline Requirements \\
\hline 1. Unified, unique, item-level identification \\
\hline 2. Decentralised management of item-level data \\
\hline 3. Generic interfaces to access item-level data \\
\hline 4. Connection of external IT-systems through open interfaces \\
\hline 5. Asynchronous messaging \\
\hline 6. Subscription mechanisms \\
\hline 7. Security mechanisms \\
\hline 8. Item-level data modelling \\
\hline 9. Transmission of decentralised data into \\
information and knowledge
\end{tabular}

Table 1: Requirements for Item-level PLM Systems

\section{IT Solutions for the Support of Item-level PLM}

In the course of an investigation of existing systems supporting item-Level PLM three environments could be identified all of which seems to fulfil the requirements listed above in section 4. These are the Electronic Product Code (EPC) in combination with the EPCglobal Framework Architecture and the PROMISE architecture. In addition an approach dealing the integration of logistics data was found (Hans, Hribernik and Thoben 2007). Although Item-level PLM is not addressed directly, a generic concept for data integration represents an important aspect while dealing with data, information and knowledge related to an item over its lifetime.

\subsection{The EPCglobal Framework Architecture}

Figure 5 shows the principle components of the EPCglobal Architecture Framework, as defined by EPCglobal (EPCGlobal Inc. 2007b). The Tag Protocol standards (UHF Class 1 and HF Generation 2) define the physical and logical requirements for RFID systems operating in the ultra-high and high frequency ranges respectively. The Low-level Reader Protocol (LLRP) deals with the standardisation of the network interface of RFID readers. The Reader Management standard is the wire protocol used by management software to monitor the operating status and health of RFID Readers. The Application Level Event standards (ALE) specify an interface through which clients may obtain filtered, consolidated RFID data whilst abstracting from the underlying physical infrastructure. The EPC Information Services (EPCIS) represents an event-based service interface for the distributed, inter-organisational sharing of application-level EPC data. The Object Name Service 
(ONS) defines a mechanism by which authoritative metadata and services associated with EPC Identifiers may be located in the network.

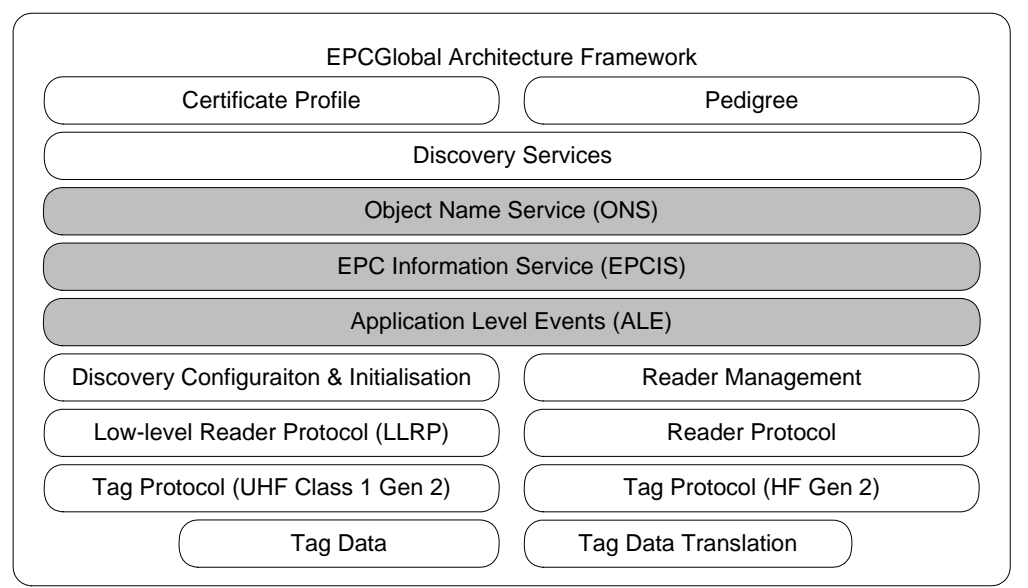

Figure 5: The EPCglobal Framework Architecture (Armenio, et al. 2007)

\subsubsection{EPC Identifiers}

The Electronic Product Code (EPC) was conceived as a means to identify all physical objects. The EPC was intended to be a short, simple and extensible code designed primarily to reference networked information. In (EPCglobal Inc 2008b), the Electronic Product Code (EPC) is described as a scheme for universally identifying physical objects. The media targeted foremost by EPC is Radio Frequency Identification (RFID) tags but other means are not excluded. The standard tag encodings consist of an EPC (or EPC Identifier) that uniquely identifies an individual object, as well as a filter value, if necessary, to enable effective and efficient tag reading

Within the EPC Identifier scheme, the concept of "Pure Identity" refers to the abstract name or number used to identify an entity, regardless of the media used to encode it, such as barcode, RFID or a database field. The Pure Identity may be represented as an Identity URI (Uniform Resource Identifier), which is a standard character representation string used to exchange identity data between system components.

\subsubsection{Application Level Events ALE}

The Application Level Event standard specifies interfaces for the communication between multiple devices and clients which exchange EPC data, and facilitates the abstraction of logical readers from the physical infrastructure (see Figure 2 below). ALE allows a client application to subscribe to a network of readers for read events. The term "readers" is not restricted to RFID readers as such, but may be interpreted as any device capable of capturing EPC data.

\subsubsection{Object Name Service ONS}

ONS is central to the second part of the EPCglobal Network architecture (EPCglobal Inc 2008a). The architecture has to ensure the accessibility of product information worldwide. The ONS utilizes the Domain Name Service (DNS) service. The DNS is required to resolve EPC codes into URLs signifying points of access to product information. Every product manufacturer that needs to offer product information through ONS has to register DNS (sub)-domains corresponding to the "Company Prefix Index" and “Object Class” that will be coded also to EPC tag as shown on the next picture.

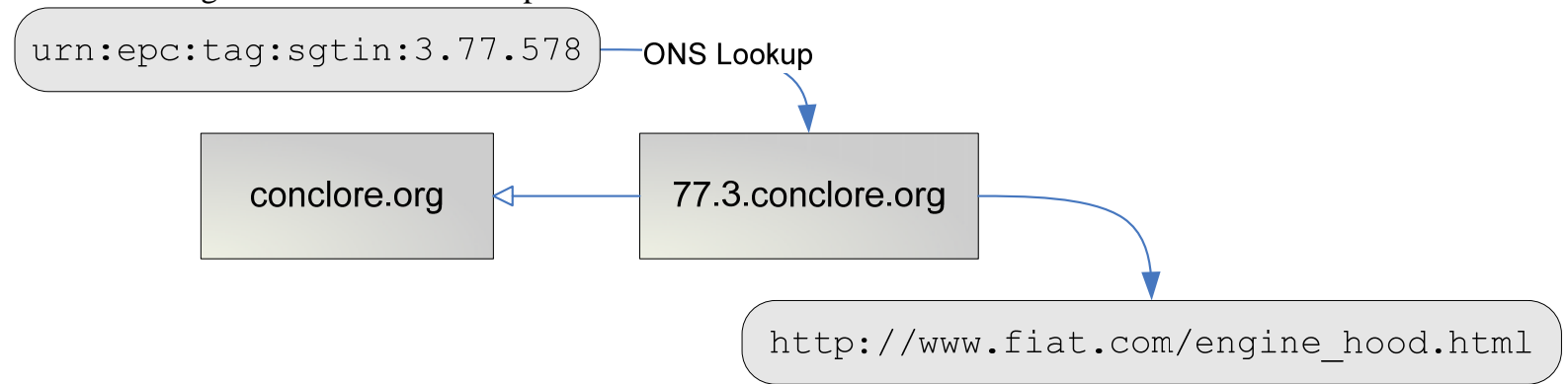

Figure 6: ONS Lookup 


\subsubsection{EPCIS}

EPCIS stands for EPC Information Services (EPCIS) and is a standard established by EPCglobal, the goal of which according to (EPCglobal Inc 2007a) is to "enable disparate applications to leverage Electronic Product Code (EPC) data via EPC-related data sharing, both within and across enterprises.” (Soon and Ishii 2007) describes its function as enabling supply chain participants in the EPCglobal network to gain real-time visibility into the movement, location and disposition of assets, goods and services throughout the world.

As shown in (Hribernik, et al. 2007), EPCIS can be leveraged to track individual products and collect, store and act upon real-time information about each. By providing a standard interface to that information, EPCIS enable cooperation partners to seamlessly query such information throughout the supply chain and product lifecycle. This leads to an increased product information transparency throughout the lifecycle, despite the individual organisations storing product-related information in distributed and heterogeneous information systems. With EPCIS, data is captured, stored and retrieved using events.

\subsection{The PROMISE Architecture}

The PROMISE Architecture as shown in Figure 7 is focussed around the concept of PEIDs (Product Embedded Information Devices). PEIDs realise the concept of smart products/components acting as embedded information gathering devices linked to sensors which are able to sense their environment and their condition wirelessly (e.g. RFID/UPnP). PEID information is communicated to backend systems via a message and event based middleware (PROMISE Data Services) using a standardised and XML based PROMISE Messaging Interface (PMI). Product Data Knowledge Modules (PDKM) integrate and manage the information from all lifecycle phases via enterprise backend systems. The decision support system (DSS) concepts allows for the implementation of decision support and control of processes such as predictive maintenance, diagnosis and analysis of use patterns.

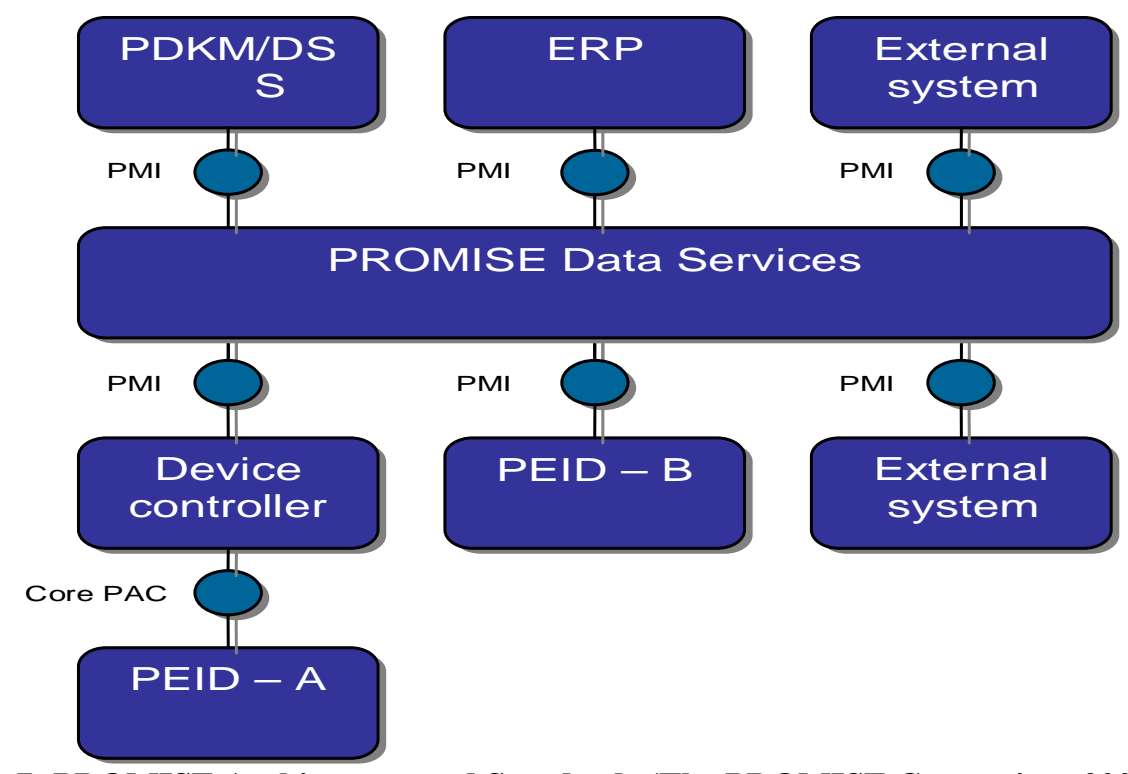

Figure 7: PROMISE Architecture and Standards (The PROMISE Consortium 2008)

\subsubsection{Product Embedded Information Devices PEID}

Product embedded information devices are embedded into a physical item or product. PEIDs can be of different categories but all PEIDs are providing item specific information. Simples ones only provide information on the product or part identification while more advanced PEIDs provide data storage capabilities, limited or medium-level computation power, sensor connectivity or data processing power. A more advanced type of PEID provides additionally functionalities such as wireless network connectivity or advance computing or data processing capabilities as provided by nowadays board computers. The PEID is a logical entity that is comprised of two portions. The first portion provides an application-independent interface and is called the Core PEID. In some applications, the Core PEID is supplemented by application-specific PEID functionality.

The Core PEID is an identification, data collection and storage system that is (at least partially) attached to products for product lifecycle monitoring and management. Note that the Core PEID does not provide 
information directly to the backend, but communicates via a Core PAC which is responsible for interfacing one or more Core PEIDs (see Figure 5).

Data may be retrieved from various types of products, ranging from small electronic articles to large, complex items like cars, locomotives etc. Due to the environmental differences in which the demonstrators operate as well as the variance in product lifecycle, cost and industry specific management requirements it is not expected that one overall Core PEID design will suit all specific requirements.

\subsubsection{Core PEID Access Container (Core PAC)}

The Core PAC is a functional representation of a collection of Core PEIDS. The Core PAC provides networking functionality to communicate with the backend layer, and implements a Device Interoperability Layer according to the above definition that the backend layer must support. Thereby, the Core PAC hides the Core PEIDs specific implementation to allow a uniform access from the backend layer. The Core PAC defines the set of functionalities that allows access to one or more PEIDs. The Core PAC contains methods to access unique product identification, and the content of one or more PEIDs in a key/value fashion, methods to store content on one or more Core PEIDs, or security features like access control on the content or parts of the content of a Core PEID collection.

\subsubsection{PROMISE Message Interface PMI}

The PROMISE Message Interface (PMI) is an XML-based standard communication protocol linking the nodes of a PROMISE architecture implementation. It provides a complete set of functionality for the access to and management of item-specific product data in an expressive and generic way, and specifies both the syntax and semantics of a request and response pairs. Its main task is to represent item-level read requests and write commands from and to PEIDs and the other nodes in the PROMISE architecture. The InfoItem-Element is the central concept of PMI, representing the message payload used to fulfil queries. InfoItems use unique identifiers to address specific items and define both application and item specific data types. PMI supports event, message and subscription based communication between all nodes and forms the backbone of the PROMISE architecture.

\subsubsection{Product Data and Knowledge Management PDKM}

The Product Data and Knowledge Management (PDKM) concept is a central component of the overall PROMISE approach, aiming at systematically integrating and managing data from all lifecycle phases of products, in particular, from design, development, production, through use and maintenance, to recycling, and finally, end of life, to support comprehensive data analysis in business intelligence application. A major challenge in realizing the PROMISE vision is the consistent integration of heterogeneous product-related data from various operational sources of the different lifecycle phases to support comprehensive data analysis. The goal is to integrate product data of the entire life cycle from different sources and furthermore to support comprehensive analysis on the integrated data and to enable the enhancement of operational businesses with the obtained insights on products. In particular, the middleware feeds the PDKM system with real-time data captured from the PEIDs attached to products, aggregations of this data or historical data maintained in proprietary field databases. The DSS depends on the data integrated and provided by the PDKM system to perform analysis tasks. The internal used data structure is a comprehensive semantic data model (SOM) for representing lifecycle data to be integrated and managed in the PDKM system. The PDKM can be assumed to potentially be of a distributed nature.

\subsection{The Semantic Mediator}

The integration of item-level PLM data from heterogeneous data sources distributed across numerous stakeholders is a key challenge. A number of predominantly semantic data integration approaches may be applied to meet this challenge. One of the major concepts in this area is the Semantic Mediator (Ullman 1997). In this approach, both syntactic and semantic descriptions of the data to be integrated are applied. The Semantic Mediator is capable of extracting knowledge regarding the data structures of the underlying data sources and subsequently transforming, decomposing and recomposing data requests according to that knowledge. The mediator relies on semantic descriptions of the data sources. In the case of item-level PLM, this implies the complete semantic modelling of product information and data across the distributed, heterogeneous sources. To this end, an approach such as the application of ontologies may be chosen (Wache 2003). 


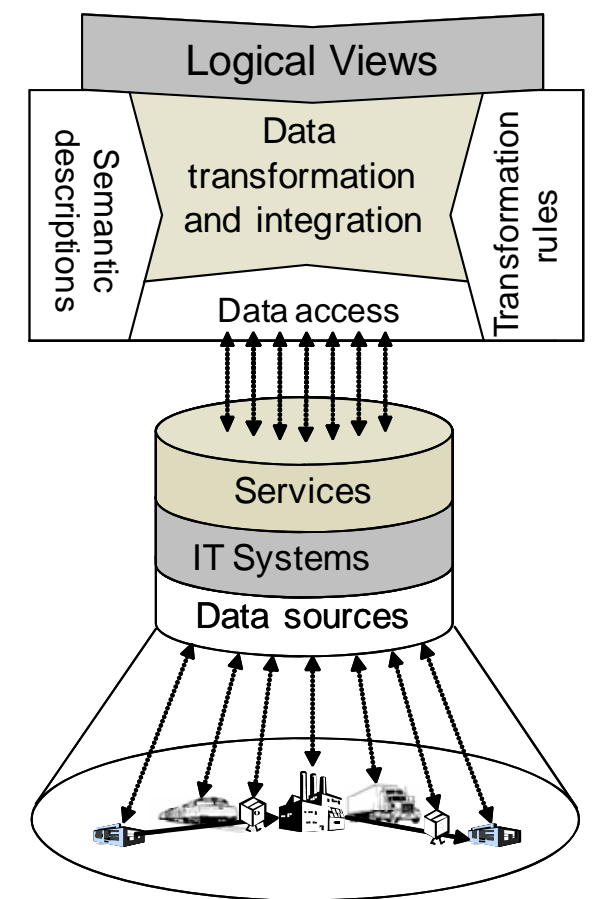

Figure 8: A Semantic Mediator Approach to Item-level PLM Data Integration

Figure 8 introduces a possible solution combining mediator technology with a service-oriented design pattern. The goal of this approach is to provide a standard interface towards freely definable logical views of item-level PLM data which may be stored in arbitrary data repositories, including mobile, dynamic sources such as sensor networks, PEIDs etc. Central to this approach is the provision of transparent mechanisms for the transformation between different standards and data structures ("data transformation and integration"), realised by means of the mediator concept. Semantic descriptions of the data sources are shown along with the necessary transformation rules by means of which the mediator is able to understand and decompose queries, forward them to the data sources in question and recompose a logical view of the returned data. Semantic modelling may be carried out using an ontology language such as the Web Ontology Language Description Logics OWL D/L (Smith, Welty and McGuinness 2004) or similar, which may be queried in using a combination of a suitable query language such as SPARQL (Prud'hommeaux and Seaborne 2008) in combination with a reasoner such as PELLET (Sirin, et al. 2007).

\section{Evaluation}

The previous sections described different approaches to implementing item-level PLM. Two of the approaches (EPCglobal Framwork Architecture and PROMISE) have been used to implement prototypical solutions for the scenarios as proof of concepts. Suitable components were selected and customised from presented approaches to specifically to solve the problems at hand. The automotive recycling scenario used components of the EPCglobal Architectural Framework to meet the requirements. The electronics recycling scenario particularly demonstrated the applicability of EPCIS to fulfil PLM tasks. The plastics recycling scenario's requirements demanded the development of components not currently covered by EPC and contributed to the creation of the PROMISE reference architecture.

Through the analysis of the scenarios presented and the resulting requirements catalogue, the authors are able to evaluate the item-level PLM approaches regarding their suitability to support reverse logistics processes. The results of the evaluation are summarized in Table 2 below.

The table reflects the degree of each the approaches' fulfilment of the requirements identified above. For the assessment, five different degrees of fulfilment have been defined. They range from no fulfilment to complete fulfilment. In the following the assessment results are discussed. 


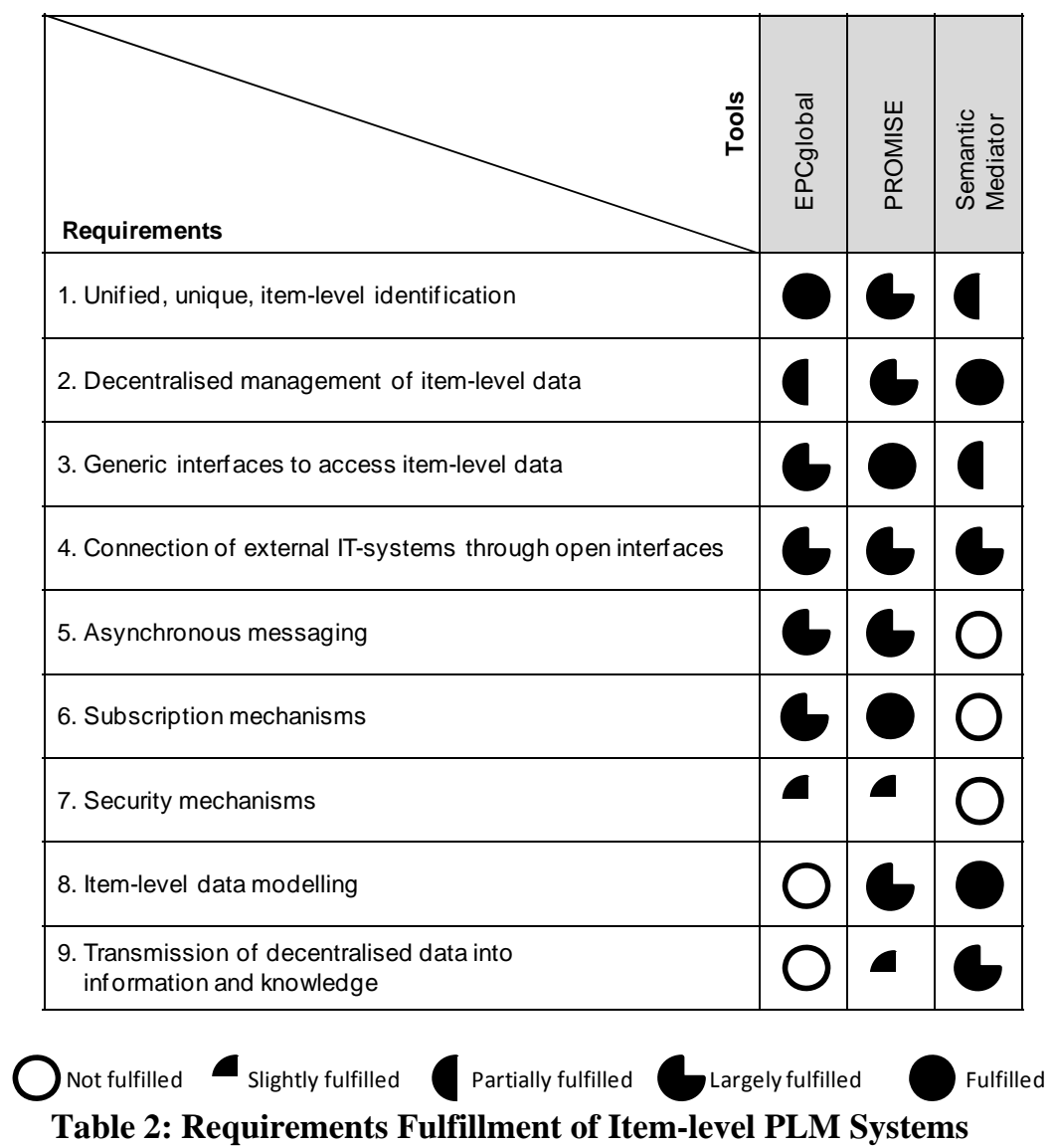

Identification can be considered as the core concern of EPC, which with its eponymous standard specifies a family of interoperable identity concepts and schemes. The PROMISE Reference Architecture makes frequent use of unique identification for the individual PEIDs and furthermore for information items in PMI. However, it refrains from specifying a proprietary identification standard. Indeed, EPC is considered a suitable option for unique identification in the PROMISE reference architecture. Whilst the PROMISE Reference Architecture and EPC both explicitly define approaches to the implementation of auto-identification in PLM solutions, the Semantic Mediator does not. As an approach with its roots mainly in the field of database integration, it is fully capable of functioning without any true item-level identification, offering a query mechanism beneath which arbitrary identification schemes such as EPC identifiers may be utilised or even combined with others. The Semantic Mediator is capable of comfortably mapping multiple identification schemes to one another via ontology mapping, so that complex product lifecycles in which different stakeholders utilise different indentifiers for the same product may benefit from this approach. For that reason, although this approach does not explicitly support unique, item-level identification, Table 2 shows partial fulfillment of requirement \#1 by the Semantic Mediator.

The Semantic Mediator explicitly addresses the decentralised management of data which is based on a semantic model of the data sources to be considered. The approach ensures a high degree of flexibility as the underlying semantic model can be modified or enhanced without the need for further modification of the surrounding IT-infrastructure. Therefore the Semantic Mediator fully satisfies this requirement. EPCIS in combination with ALE and ONS support a decentralised storage and retrieval of item level data but the concept is very much event-driven. Due to its focus on logistics, PLM-specific aspects like the continuous storage of item-level data (e.g. in form of operational field data) are not fully supported. The PROMISE Reference Architecture achieves a large fulfilment level here as PDKM explicitly addresses the integration and management of item-level data reflecting a products' lifecycle. As for the Semantic Mediator, semantic descriptions of the data form the basis for their management. In contrast to the Semantic Mediator these descriptions are static. Therefore changes here requires significant effort in order to update the software components which are reflected (e.g. database schemes).

With its ONS component, EPCglobal largely fulfils the requirement concerning generic interfaces supporting the access of item-level data. Each EPC refers to a set of services offering specific functionality which is associated the item the EPC is belonging to. Due to its focus on logistics, it cannot fully reflect the PLM context. In contrast to EPCglobal, PROMISE follows a message based approach. The reference architecture specifies a 
generic set of messages reflecting the specific requirements for the exchange of PLM data. As a service-based approach the Semantic Mediator also provides a generic approach for accessing item-level data. In contrast to the other concepts it lacks an integrated concept supporting a unified, unique identification of individual items. Therefore the answer of a services request requires further processing to isolate the data of interest.

All of the approaches achieve the same level of fulfilment of the requirement concerning the connection to external IT-systems. Here the PROMISE reference architecture follows the path of conventional enterprise application integration approaches by proposing a messaging format for the communication between its components. EPCglobal offers the open EPCIS specification forming the base for the integration with other ICT environments. A connection to external IT-systems can be established by offering suitable services which are delivered as the result of an ONS lookup procedure. The Semantic Mediator may be complimented by a set of generic services which can be integrated into external IT systems. These services may also adhere to an open specification; integration into $3^{\text {rd }}$ party systems requires a similar effort to EPCglobal or PROMISE.

Concerning support for asynchronous messaging, PMI defines an XML message format along with specific operations, including asynchronous communication between the entities involved in PLM scenarios. In this context EPCglobal supports asynchronous communication through its event subscription mechanisms. Neither the PROMISE nor EPCglobal architectures expects the sender of a message or event to halt operations until an the receipt of an subscription response. The Semantic Mediator neither natively offers message-based communication nor asynchronous service calls. The same holds true for subscription mechanisms which are not supported. In this context EPCglobal explicitly offers the possibility to subscribe certain events. Due to the focus on logistics these subscriptions only partially reflect PLM events. In contrast to that the PROMISE PMI directly support the subscription of PLM-events.

Although product and process data are nowadays considered as a valuable resource of companies especially for the manufacturing industry - none of the approaches discussed here explicitly provides specific means or concepts to ensure security.

The remaining two requirements focus on semantics and the transmission of data into information and knowledge. Only PROMISE and the Semantic Mediator explicitly offer semantic descriptions. Thus, item-level data modelling is supported only by these approaches. For the PROMISE architecture the products to be dealt with are specified using the Semantic Object Model (SOM) which is used for the generation of internal structures e.g. within the PDKM. The SOM can be considered as a structural description of a product reflecting its attributes and associated data types. The Semantic Mediator requires detailed descriptions for each of the data sources which should be integrated. These descriptions have to be exhaustive enough to support an automatic data transformation. Thus these models can be considered as more comprehensive than for PROMISE.

Concerning the transmission of item-level data into information and knowledge the Semantic Mediator seems to offer more potential than the PROMISE architecture. This is because the object model of PROMISE mainly addresses the structure of the data to be captured for a product. In contrast to that the Semantic Mediator is able to transform different data elements solely based on the available semantic descriptions. These descriptions may be further exploited for the generation of information and knowledge related to a products state within its lifecycle.

\section{Summary and Conclusions}

By analyzing several business cases it was shown, that item-level PLM can be applied to support reverse logistics processes. Different approaches to that end were presented. One of them (PROMISE) directly addresses item-level PLM. Another (EPCglobal) represents an industrial standard which enjoys widespread acceptance but is very much focused on the field of logistics and SCM. Finally, the Semantic Mediator is an approach which explicitly addresses the problem of data integration. In order to assess these approaches regarding their applicability for an item-level PLM solution to improve reverse logistics processes, a number of key requirements were identified. The assessment of the approaches has shown that the former two provide adequate support for item-level PLM in the field of reverse logistics by fulfilling the majority of the identified requirements. The latter exhibits very strong benefits in very specific areas but cannot be considered as balanced and general as the first two, ruling the approach out as a sole component of an IT solution supporting item-level PLM for reverse logistics.

Considering the specific strengths and weaknesses of all the three solutions, application areas can be identified which appear more suitable to each of the approaches, and different combinations of the individual approaches' components may be considered to build solutions for each of these areas.

The focus of EPCglobal Framework Architecture is on item-level logistics sector and covers a wide range, but not all of the requirements with respect to PLM in the field of reverse logistics. On the other hand, EPC is an international standard enjoying widespread adoption in the field of identification and logistics. Other components of the EPCglobal Framework Architecture such as EPCIS support integration into other enterprise 
systems. Consequently, sustainability applications where EPCglobal infrastructures or standards are already in use and which logistics or SCM processes are already tracked using the architecture may benefit strongly from using it as the basis of an item-level PLM approach.

However, the PROMISE architecture explicitly focuses the application area of item-level PLM. Therefore, the underlying reference architecture addresses core PLM issues such as the subscription of item-level data or a powerful messaging system specifically for item-level PLM, and is the only approach to explicitly model itemlevel data from a PLM perspective. For this reason, the PROMISE architecture may be considered as good starting point for the implementation of a sustainability solution which is to be developed from scratch and has high demands towards item-level PLM support.

The Semantic Mediator is a powerful solution in the area of data modeling, the transmission of data into information and knowledge, and data integration. This is highly useful in sustainability applications which address a large number of fluctuating stakeholders along with corresponding data sources. In such cases, this approach should be taken into account. However, the lack of any true support for item-level PLM makes the Semantic Mediator viable only in the context of wider architecture considerations. Due to its very focused strengths in the area of data integration, it is worthwhile considering its application as an supplement to either of the more general EPCglobal or PROMISE approaches.

\section{Acknowledgments}

Parts of this work were carried out in the EU FP6 projects CONCLORE (Controlled Closed Loop Recycling for Life-Cycle Optimised Industrial Production), PROMISE (Product Lifecycle Management and Information using Smart Embedded Systems) and the DFG-funded Collaborative Research Center 637 Autonomous Cooperating Logistics Processes - A Paradigm Shift and its Limitations Subproject C2 Data Integration. The authors gratefully acknowledge the contributions of these projects' consortia and funding organizations to the research presented in this paper.

\section{References}

Abramovici, M, and O Sieg. "PDM - Technologie im Wandel - Stand und Entwicklungsperspektiven. Orientierung für die Praxis.” Industrie Management, 2001.

Ameri, F, and D Dutta. "Product Lifecycle Management - Closing the Knowledge Loops.” Computer-Aided Design and Applications, 2005.

Armenio, Felice, et al. The EPCglobal Architecture Framework. Lawrenceville, New Jersey: EPCglobal Inc, 2007.

EPCglobal Inc. EPC Information Services (EPCIS) Version 1.0.1 Specification. Lawrenceville, New Jersey: EPCglobal Inc, 2007a.

EPCglobal Inc. EPCglobal Object Name Service (ONS) 1.0.1. Lawrenceville, New Jersey: EPCglobal Inc, 2008a.

EPCglobal Inc. EPCglobal Tag Data Standards Version 1.4. Lawrenceville, New Jersey: EPCglobal Inc, $2008 \mathrm{~b}$.

EPCglobal Inc. The Application Level Events (ALE) Specification, Version 1.1.1.Part I: Core Specification. EPCglobal Ratified Standard with Fixed Errata, Lawrenceville, New Jersey: EPCglobal Inc., 2009.

EPCGlobal Inc. The EPCglobal Architecture Framework. Standard Specification, EPCGlobal Inc., $2007 \mathrm{~b}$.

Fowler, Julian. STEP for Data Management, Exchange and Sharing. UK: British Library, 1995.

Främling, Kary, Mark Harrison, and James Brusey. "Globally Unique product Identifiers - Requirements and Solutions to Product Lifecycle Management.” Information Control Problems in Manufacturing: Proceedings Volume from the 12th IFAC International Symposium. ISBN 978-0080446547: Elsevier B.V., 2007. 855-860.

Hans, Carl, Karl Hribernik, and Klaus-Dieter Thoben. “An Approach for the Integration of Data within Complex Logistics Systems.” LDIC2007 Dynamics in Logistics: First International Conference. Proceedings. Heidelberg: Springer, 2007. 381-389.

Hribernik, Karl A., Martin Schnatmeyer, Andreas Plettner, and Klaus-Dieter Thoben. “Application of the Electronic Product Code EPC to the Product Lifecycle of Electronic Products.” RFID Convocation. Brussels, Belgium: European Commission, 2007. 
Hribernik, Karl Anthony, Lutz Rabe, Robertino Solanas, and Klaus-Dieter Thoben. "Conception of Architecture to Support Item-specific Product Information Management." 5th International Conference on Product Lifecycle Management. Seoul, Korea, 2008.

Jun, Hong-Bae, Dimitris Kiritsis, and Paul Xirouchakis. "Research Issues on Closed-loop PLM.” Computers in Industry, December 2007: 855-868.

Kahn, O, et al. „RFID in automotive: a closed-loop approach.“ Herausgeber: Klaus-Dieter Thoben, Kulwant S. Pawar, Marco Taisch und Sergio Terzi. 12th International Conference on Concurrent Enterprising. Milan, Italy: Centre for Concurrent Enterprise, 2006.

Kärkkäinen, M, Kary Främling, and T Ala-Risku. "Intergrating Material Flows Using a Distributed Peer-to-Peer Information System.” Edited by Harinder S Jagdev, Hans Wortmann, H.-J. Pels and A Hirnscall. Proceedings of APMS Conference. Eindhoven, Netherlands, 2002. 463-473.

Kärkkäinen, Mikko, Jan Holmström, Kary Främling, and Karlos Artto. "Intelligent Products - A Step Towards a More Effective Project Delivery Chain.” Edited by J C Wortmann. Computers in Industry (Elsevier) 50, no. 3 (February 2003): 41-151.

Kiritsis, Dimitris, Ahmed Bufardi, und Paul Xirouchakis. „Research issues on product lifecycle management and information tracking using smart embedded systems.“ Herausgeber: Jay Lee und Jun Ni. Advanced Engineering Informatics (Elsevier Ltd.) 17, Nr. 3-4 (July-October 2003): 189-202.

Prud'hommeaux, Eric, and Andy Seaborne. SPARQL Query Language for RDF. W3C Recommendation, World Wide Web Consortium, 2008.

Schuh, Günther, Achim Kampker, Bastian Franzkoch, und Nils Wemhöner. Studie Intelligent Maintenance Potentiale Zustandsorientierter Instandhaltung. Study, Essen, Germany: ifm electronic GmbH, 2005.

Sirin, Evren, Bijan Parsia, Bernardo Cuenca Grau, Aidtya Kalyanpur, and Yarden Katz. "Pellet: A Practical OWL-DL Reasoner." Journal of Web Semantics - Web Semantics: Science, Services and Agents on the World Wide Web, April 2007: 51-33.

Smith, Michael K, Chris Welty, and Deborah L McGuinness. OWL Web Ontology Language. W3C Recommendation, World Wide Web Consortium, 2004.

Soon, Tan Jin, and Shin-ichii Ishii. "EPCIS and Its Applications." Synthesis Journal, 2007: 109-124.

Stark, John. Product Lifecycle Management. Berlin, Germany: Birkhäuser, 2004.

The PROMISE Consortium. PROMISE Architecture Series Volume 1: Architecture Overview. Finland: Promise Innovation Oy, 2008.

Ullman, J D. „Information Integration Using Logical Views.“ Herausgeber: F N Afrati und P Kolaitis. Proceedings of the 6th International Conference on Database Theory ICDT '97. 1997.

Ventä, Olli. Intelligent Products and Systems. Technology Theme - Final Report. VTT, Espoo: VTT Publications, 2007, 304 s.

Wache, Holger. Semantische Mediation für heterogene Informationsquellen. Vol. Dissertationen zur Künstlichen Intelligenz Band 261. Berlin, Germany: Akademische Verlagsgesellschaft Aka GmbH, 2003.

Wong, C Y, Duncan McFarlane, A Ahmad Zaharudin, and V Agarwal. "The Intelligent Product Driven Supply Chain.” Proceedings of IEEE International Conference on Systems, Man and Cybernetics, 2002. Tunisia: IEEE, 2002. 